\title{
Assets em Áreas Protegidas: Estudo de Caso em Áreas Úmidas
}

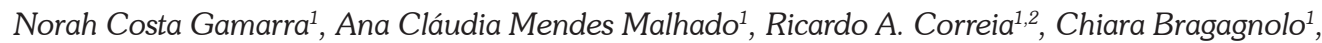 \\ João V. Campos-Silva ${ }^{1}$, Paul Jepson ${ }^{3}$ \& Richard James Ladle ${ }^{1}$
}

Recebido em 30/01/2018 - Aceito em 27/09/2018

RESUMO - As áreas protegidas (APs) são as principais ferramentas capazes de assegurar a preservação dos sistemas naturais e sua respectiva biodiversidade, principalmente no caso de áreas com prioridade para conservação, como ocorre com as áreas úmidas. A implementação de APs tem potencial para gerar benefícios fundamentais para proteger paisagens icônicas, espécies ameaçadas e serviços ecossistêmicos; entretanto, essas áreas têm custos sociais e econômicos que, muitas vezes, se tornam difíceis de justificar em períodos de crescente insegurança alimentar e crise financeira. Recentemente, sugeriu-se um modelo conceitual para apoiar a gestão das APs (PA Asset Framework) que (re)define as APs como um sistema de assets biofísicos, humanos, de infraestrutura, institucionais e culturais. De acordo com esse marco, as APs podem ser geridas e planejadas para gerar diferentes formas de valores, desse modo contribuindo para aumentar sua resiliência política/social e identificar investimentos financeiros que possam auxiliar o reconhecimento $e$ a captura de valores por distintos públicos. Neste artigo, aplicamos o PA asset framework para levantar a presença de assets em parques nacionais localizados em áreas úmidas, a partir da revisão sistemática dos planos de manejo. Nestes, identificamos os assets presentes e mais recorrentes em tais áreas. Nossos resultados mostram que o número de assets representados e reconhecidos nos planos de manejo é limitado, concentrando-se, principalmente, em algumas classes biofísicas relacionadas à conservação da biodiversidade (ex: espécies de importância para a conservação e espécies de importância econômica), de infraestrutura necessária para manutenção das atividades de gestão (ex: funcionário permanente, infraestrutura para gestão, eletricidade e veículos) e institucionais (estratégias de planejamento e zoneamento). Por outro lado, dada a importância sociocultural das áreas úmidas em nível nacional e global, o baixo reconhecimento de assets humanos e culturais nessas áreas sugere que a inclusão desses valores nos planos de manejo pode ser benéfica para potencializar os valores associados a essas tipologias de asset. Argumentamos que o levantamento dos assets em APs, além de revelar os bens/recursos/atributos/dimensões-chave que podem estar sendo negligenciados nos planos de manejo, oferece uma abordagem inovadora para que os gestores possam elaborar estratégias de investimento a partir do reconhecimento de valores tangíveis e intangíveis das APs e dos seus assets, para além dos biofísicos.

Palavras-chave: Recursos e valores; manejo; unidades de conservação.

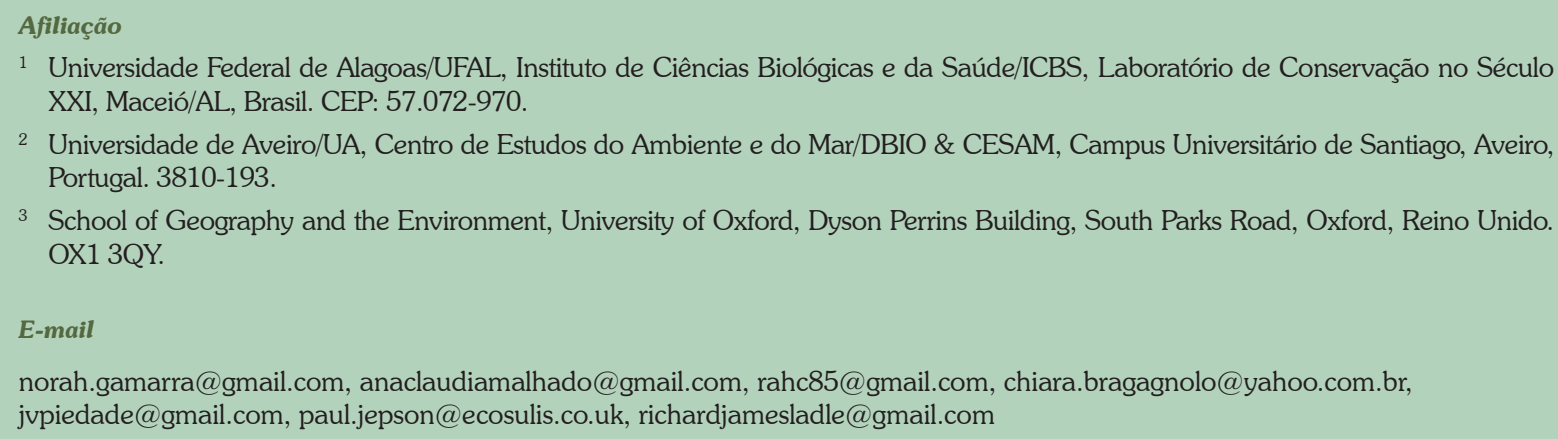


ABSTRACT - The Protected Areas ASSETS: Case Study in Wetlands. Protected áreas (PAs) are globally recognized as the main mechanism to ensure the conservation of natural systems and their biodiversity, namely in high conservation priority areas such as wetlands. Although PAs are essential to secure support for the protection of iconic landscapes, threatened species and ecosystem services, they often have important social and economic costs that are increasingly difficult to justify in periods of economic and food insecurity. A recently proposed conceptual model to support PA management (re-)defines these areas as a system of biophysical, human, infrastructure, institutional and cultural assets. According to this model, PAs can be managed and planned to generate different forms of value for society, thus contributing to increase their social and political resilience and facilitating the identification of potential investments that can help to identify and capture value by multiple sectors of the society. In this study, we apply the PA asset framework to characterize the presence of assets in Brazilian National parks located in wetlands through the systematic revision of their management plans. Specifically, we identify which assets are present and most frequently identified in these areas. Our results suggest that the number of identified assets in management plans is limited, focusing mostly on biophysical assets associated with biodiversity conservation (e.g. species of conservation importance, economically valuable species) and the infrastructure necessary for park management and maintenance activities (e.g. permanent workers and managers, management infrastructure, electricity and vehicles, and planning and zoning strategies). On the other hand, given the national and global socio-cultural importance of wetlands, a lack of recognition of human and cultural assets suggests that their inclusion in management plans may benefit their value generating potential. In summary, we argue that identifying PA assets and revealing their benefits and values, which may be neglected in management plans, offers an innovative approach for managers to explore new investment and management opportunities for value generation in PAs.

Keywords: Resources and values; management; conservation units.

RESUMEN - Assets en Áreas Protegidas: Estudio de Caso en Humedales. Las áreas protegidas (AP) son reconocidas mundialmente como el principal mecanismo para garantizar la conservación de los sistemas naturales y su biodiversidad, principalmente en áreas de alta prioridad de conservación, como los humedales. Estas son esenciales para asegurar el apoyo para la protección de paisajes icónicos, especies amenazadas y servicios de los ecosistemas, a menudo tienen importantes costos sociales y económicos que son cada vez más difíciles de justificar en períodos de inseguridad económica y alimentaria. Un modelo conceptual recientemente propuesto para respaldar la gestión de AP (re)define estas áreas como un sistema de activos biofísicos, humanos, de infraestructura, institucionales y culturales. De acuerdo con este modelo, las AP pueden ser manejadas y planeadas para generar diferentes formas de valor para la sociedad, contribuyendo así a aumentar su resiliencia social y política y facilitando la identificación de posibles inversiones que pueden ayudar a identificar y capturar el valor de múltiples sectores de la sociedad. En este estudio, aplicamos el cuadro teórico de los activos para caracterizar la presencia de activos en los parques nacionales brasileños ubicados en humedales mediante la revisión sistemática de sus planes de manejo. Específicamente, identificamos cuales activos están presentes y se identifican con mayor frecuencia en estas áreas. Nuestros resultados sugieren que la cantidad de activos identificados en los planes de manejo es limitada, centrándose principalmente en los activos biofísicos asociados con la conservación de la biodiversidad (por ejemplo, especies de importancia para la conservación, especies económicamente valiosas), la infraestructura necesaria para el manejo y las actividades de mantenimiento del parque (por ejemplo, trabajadores permanentes, infraestructura de gestión, electricidad y vehículos) $e$ institucionales (estrategias de planificación y zonificación). Dada la importancia sociocultural nacional y global de los humedales, la falta de reconocimiento de activos como los recursos humanos y culturales sugiere que su inclusión en los planes de manejo puede beneficiar su potencial de generación de valor. En resumen, argumentamos que identificar los activos de AP y revelar sus beneficios y valores ofrece un enfoque innovador para que los gestores exploren nuevas inversiones y oportunidades de gestión para la generación de valor tangible e intangible en las AP.

Palabras clave: Recursos y valores; gestión; unidades de conservación.

\section{Introdução}

Embora fundamentais para as sociedades humanas, os ambientes aquáticos têm se tornado os ecossistemas mais ameaçados globalmente, com taxas de perda de espécies substancialmente maiores que as observadas em ambientes terrestres (Ricciard et al. 2009, Sala et al. 2010). Os vários 
mecanismos nacionais e internacionais para assegurar a preservação dos sistemas naturais aquáticos e para deter o declínio contínuo da biodiversidade são de extrema importância (Kleijn et al. 2014), pois a conservação desses ambientes, incluindo seus serviços ecossistêmicos e o bem-estar das populações tradicionais que nele residem, configura-se como um dos grandes objetivos do milênio (ONU 2015, Russi et al. 2013).

Entre eles, em vigor desde 1975, a Convenção de Ramsar tem por finalidade proteger zonas úmidas de importância internacional - de caráter representativo ou singular; com espécies ameaçadas ou endêmicas e de importância para manutenção da biodiversidade; e importantes para populações de aves aquáticas - estabelecendo marcos para ações nacionais e para a cooperação entre os países signatários (Scott \& Jones 1995, Ramsar Convention Secretariat 2013). É a única convenção global intergovernamental que aborda as interações entre a água e os ecossistemas (MEA 2005), tendo como base três pilares: i) o uso racional das áreas úmidas; ii) a atenção especial às áreas úmidas de importância internacional; e iii) a cooperação internacional (Ramsar Convention Secretariat 2013).

No Brasil, o Comitê Nacional de Zonas Úmidas (CNZU) é responsável pela tomada de decisões e definição de diretrizes para a implementação da Convenção de Ramsar. Instituído pelo Decreto s/n, de 23 de outubro de 2003, e composto por diferentes segmentos, órgãos, entidades e organizações não governamentais, o CNZU atua na definição de novas áreas úmidas prioritárias para conservação, seu planejamento e gestão (MMA 2015). O comitê, atendendo às premissas da Convenção de Ramsar, elabora e recomenda a adoção de um sistema de classificação brasileiro, além de propor a seguinte definição de áreas úmidas:

"[...] ecossistemas na interface entre ambientes terrestres e aquáticos,
continentais ou costeiros, naturais ou artificiais, permanente ou
periodicamente inundados ou com solos encharcados. As águas
podem ser doces, salobras ou salgadas, com comunidades de
plantas e animais adaptados à sua dinâmica hídrica" (MMA 2015).

A preocupação com essas áreas em particular se deve às suas especificidades, aos serviços ambientais que proporcionam, aos seus altos níveis de diversidade e produtividade e seu amplo valor econômico, ecológico e social (Amano et al. 2017, Chaikumbung et al. 2016), incluindo sua importância na manutenção da biodiversidade e de regimes hidrológicos; na provisão de água potável, madeira e produtos não madeireiros, plantas medicinais e outros recursos de alta importância para as comunidades tradicionais que habitam seus domínios (Junk et al. 2013, MEA 2005).

Estratégias eficientes de conservação são imperativas para essas áreas (Junk et al. 2013, MMA 2015). As áreas protegidas (APs) têm sido a ferramenta global mais difundida de conservação da biodiversidade e dos sistemas naturais (Watson et al. 2014). Até 2017, no Brasil, as áreas úmidas compreendiam 22 unidades de conservação $(\mathrm{UCs})^{1}$ nas esferas federal e estadual, inclusas também três reservas particulares do patrimônio natural (RPPNs). Deste total, oito são parques nacionais (PARNAs), categoria mundialmente reconhecida, bem consolidada e representada em território brasileiro (Bragagnolo et al. 2016, Correia et al. 2018).

Muitos PARNAs começaram como santuários de vida selvagem ou monumentos naturais e faziam parte de uma estratégia de "construção de uma nação" adotada por muitos governos, especialmente em países recentemente democratizados (Ladle, Jespson \& Gillson 2011). Hoje, entretanto, o aumento

1 Ver mais em: Ramsar Sites Information Service (https://rsis.ramsar.org). 
populacional e a pressão para conversão de áreas naturais para agricultura, geração de energia $e$ infraestrutura, por exemplo, fazem com que essas áreas passem, em alguns casos, a ser vistas como barreiras ao desenvolvimento socioeconômico (Foley 2005, Watson et al. 2014).

Essa perspectiva lança as APs em um cenário conflituoso, resultando em lacunas em seus orçamentos (Oliveira \& Bernard 2017), redução de pessoal (Watson et al. 2014) e aumento em ações de redução, extinção ou reclassificação de áreas protegidas (em inglês, conhecido como PADDD - Protected Area Downsizing, Downgrading or Degazettement) (Bernard, Penna \& Araújo 2014; Mascia et al. 2014, Pack et al. 2016, Symes et al. 2016). As APs, portanto, vêm perdendo cada vez mais espaço, com, por exemplo, governos descumprindo compromissos internacionais (Swenson et al. 2011) e com o enfraquecimento da aplicação legal de regulamentos ambientais, frente a uma instabilidade política (Amano et al. 2017).

\section{Como assegurar a manutenção de áreas protegidas?}

Diante das crescentes dificuldades que ameaçam o funcionamento das APs, oportunizar novas fontes de investimento e aumentar o apoio político em APs e, consequentemente, em áreas úmidas, se torna emergencial. Jepson e colaboradores (2017) argumentam que enquadrar APs como uma forma especial de ativos (assets, em inglês), de modo que os seus valores possam ser ampliados para além do valor intrínseco da conservação da natureza e possam incluir a ampla variedade de benefícios que eles proporcionam para a sociedade em geral, poderia fornecer uma abordagem útil para gerar investimento e fortalecer a resiliência política e social dessas áreas (Caldecott \& Jepson 2014; Vucetich, Bruskotter \& Nelson 2015).

Nesse contexto, a identificação de assets e valores tangíveis e intangíveis presentes nas APs seria essencial para o desenvolvimento de estratégias que garantissem a conservação dos recursos naturais, a geração de renda e/ou qualidade de vida aos usuários e a atração de investimentos de diferentes setores (Caldecott \& Jepson 2014).

\section{Assets em áreas protegidas}

O termo "asset" é amplamente utilizado em economia e finanças, representando bens, propriedades, títulos ou recursos que podem gerar valor, serem comprados, transferidos ou compartilhados (Jepson et al. 2017). Pensando no conceito para as APs e sistemas naturais, os assets podem ser definidos como bens ou recursos que podem ser protegidos, manejados e investidos para gerar valor (monetário ou não) que podem ser capturados por grupos da sociedade, em um contexto socioecológico (Jepson et al. 2017). Os assets naturais de APs são aqui classificados em termos biofísicos, humanos, de infraestrutura, institucionais e culturais (Figura 1, Quadro 1) (Caldecott e Jepson 2014, Jepson et al. 2017).

O marco de identificação e manejo dos assets proposto por Jepson e colaboradores (2017) é baseado em três componentes principais: i) os assets da área protegida; ii) as formas de valor geradas por esses assets; e iii) os domínios da sociedade em que os beneficiários podem capturar o valor dos assets da área protegida.

$\mathrm{O}$ valor dos assets depende das interações entre elementos naturais e o componente humano existente no sistema socioecológico; essas interações geram diferentes combinações de valor que poderão ser capturadas por diferentes grupos em diferentes domínios da sociedade (Figura 2). Por exemplo, a complexidade dos recifes coralíneos do PARNA Abrolhos (ver Quadro A) só se torna um asset biofísico de "beleza cênica" quando interage com culturas que valorizam tal paisagem. O ponto importante é que o valor de cada asset decorre de práticas de envolvimento entre a sociedade humana com a natureza (e APs) e, portanto, pode assumir diferentes formas em diferentes contextos socioculturais - como é colocado, para as áreas úmidas, no documento de Serviços Ecossistêmicos da Secretaria da Convenção de Ramsar 
(2009a): "As paisagens de zonas úmidas e a vida selvagem que valorizamos hoje resultam tipicamente de interações complexas entre as pessoas e a natureza ao longo dos séculos", tendo em vista que a gestão de paisagens naturais e da biodiversidade parte de uma expressão cultural, que, por sua vez, molda a cultura humana (Papayannis \& Pritchard 2008).

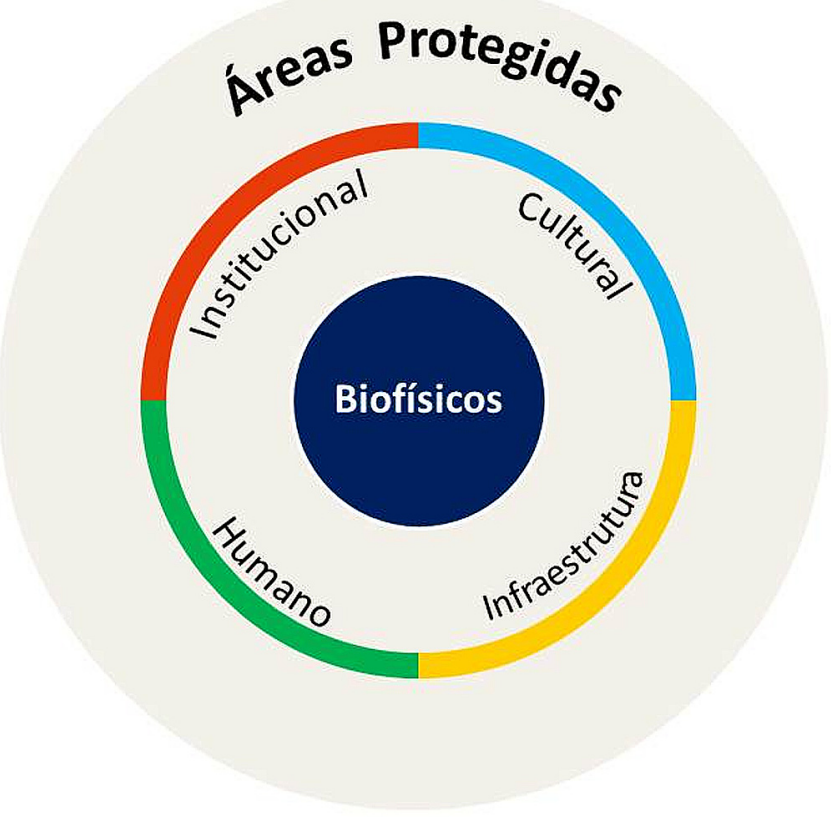

Figura 1 - Assets de áreas protegidas. (Figura adaptada de Caldecott \& Jepson 2014).

Quadro 1 - Exemplificação das categorias de assets.

\begin{tabular}{|l|l|}
\hline Biofísicos & $\begin{array}{l}\text { Atributos bióticos e abióticos presentes dentro dos limites de uma AP. Eles incluem beleza } \\
\text { cênica, espetáculos naturais, espécies icônicas e raras, a diversidade de espécies, os recursos } \\
\text { naturais e as funções do ecossistema. }\end{array}$ \\
\hline Humanos & $\begin{array}{l}\text { Formas de conhecimento incorporadas a uma AP que permitem a conservação de bens } \\
\text { biofísicos e a captura de valor também de outros assets. Exemplo: funcionários do parque, } \\
\text { população, pesquisadores, visitantes e voluntários regulares. }\end{array}$ \\
\hline Infraestrutura & $\begin{array}{l}\text { Instalações construídas e materiais que permitem a geração de valor: estradas de acesso, } \\
\text { teleféricos, trilhas, equipamentos de trabalho, hotéis, centros de visitantes etc. }\end{array}$ \\
\hline Institucionais & $\begin{array}{l}\text { Relações organizacionais, jurídicas e de gestão que constroem uma AP. Incluem projetos, órgão } \\
\text { de gestão e plano, acordos comunitários e próprio documento de plano de manejo em si. }\end{array}$ \\
\hline Culturais & $\begin{array}{l}\text { Expressões que compõem o perfil de uma AP e a história da área. Como, por exemplo, } \\
\text { imagens icônicas, interpretações artísticas, conhecimento tradicional dos povos, registros } \\
\text { arqueológicos etc. }\end{array}$ \\
\hline
\end{tabular}

Os valores dos assets podem ser traduzidos em um "estoque" de recursos renováveis, serviços ecossistêmicos ou biodiversidade, através de investimentos no desenvolvimento de assets humanos associados (por exemplo: cientistas de recursos, engenheiros florestais), infraestrutura (por exemplo: estradas, viveiros) e assets institucionais (por exemplo: regulamentos, certificação). 


\section{Formas de valor}

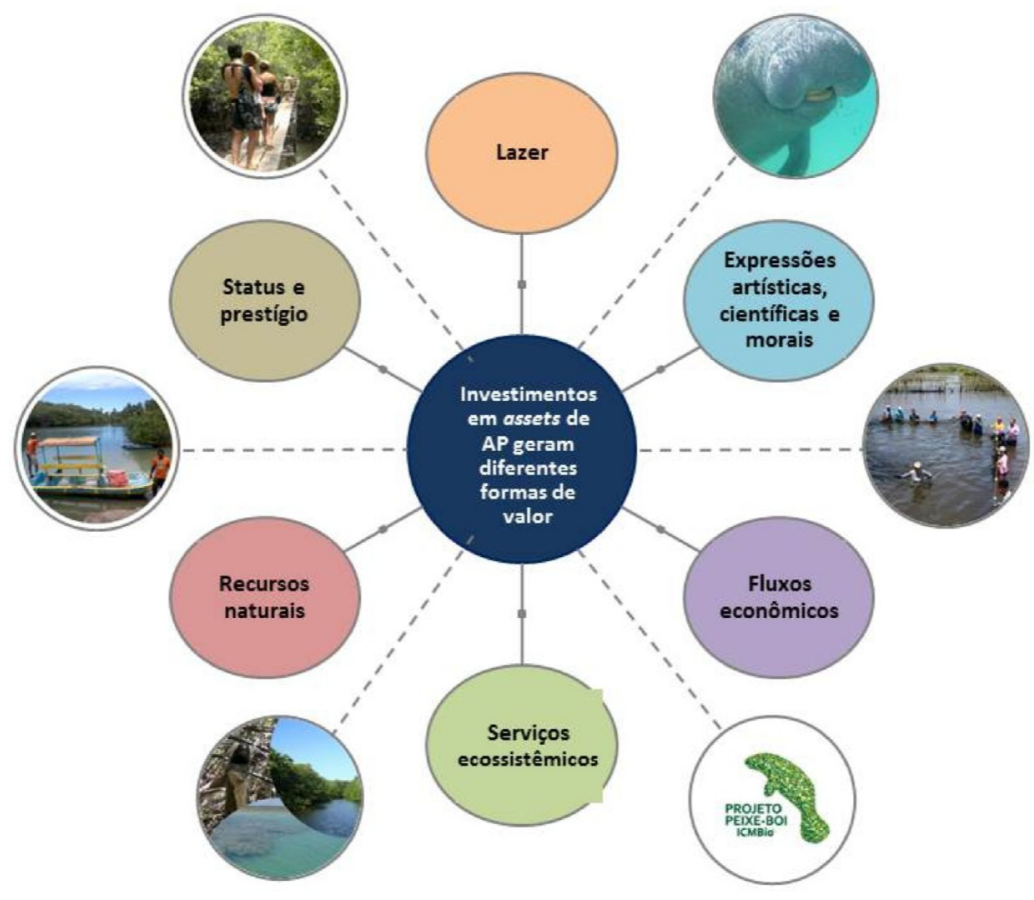

Captura de valor

Para nações, retorna sob a forma de senso de identidade regional, saúde pública, reconhecimento dos direitos de povos e comunidades tradicionais, gestão ambiental e presença em regiões remotas

Para cidadãos, uma variedade de retorno, como expressões artísticas, espirituais, científicas, recreação de aventura, bem-estar e qualidade de vida

Figura 2 - Criação de valor em áreas protegidas. Figura adaptada de Caldecott \& Jepson 2014. (Todas as imagens utilizadas estão disponíveis no site do ICMBio, na página da APA Costa dos Corais e da Associação Peixe Boi).

Esse enquadramento apresenta quatro domínios principais da sociedade, em que o valor da área protegida pode ser gerado e capturado: i) cidadãos; ii) organizações e profissões; iii) economias e empresas; e iv) nações e políticas (Figura 2). As práticas culturais em cada um desses domínios interagem com as APs para gerar formas de valor para a pessoa, o grupo ou a entidade envolvida. Por exemplo, os cidadãos capturam valores de qualidade de vida, como expressão 
estética, sensação de bem-estar físico de assets biofísicos, como a beleza cênica e espécies icônicas. Esses valores podem ser capturados através de práticas tais como trekking ou exibição de documentários sobre a vida selvagem.

A estrutura de avaliação de assets de APs, portanto, destina-se a ser uma ferramenta heurística para aumentar a eficiência da gestão das APs e dos investimentos em valor natural a longo prazo. Busca-se mudar o foco da conservação para otimizar como os benefícios de instrumentos de conservação podem ser melhor aproveitados (Kleijn et al. 2014). Considerando, também, o aumento da população humana e sua dependência da água e das áreas úmidas, reforça a urgência de um reconhecimento pleno dos valores e benefícios gerados pela natureza (Russi et al. 2013).

Neste contexto, o presente artigo tem como objetivo apresentar o PA asset framework a partir da sua aplicação para identificação de assets em PARNAs brasileiros em áreas úmidas.

\section{Metodologia}

\section{Levantamento de assets nos parques nacionais}

Para o levantamento de assets em cada PARNA, utilizamos a lista proposta por Jepson et al. (2017) adaptada por Gamarra (veja Gamarra (2017) e Gamarra et al. (2019)). Baseados em uma lista preliminar de 101 tipos de assets subdivididos em 40 subcategorias que, por sua vez, se enquadram nas cinco grandes categorias identificadas anteriormente (Quadro 1), fizemos uma análise documental e de conteúdo (Berelson 1952, Bardin 1977) nos planos de manejo dos parques nacionais das áreas úmidas brasileiras para compilar a ocorrência de assets em cada parque, utilizando apenas as informações contidas nos documentos.

Os planos de manejo (PMs) foram escolhidos por serem documentos padronizados, que mostram um diagnóstico detalhado dos sistemas naturais presentes nas APs. Além disso, os PMs trazem detalhes sobre o planejamento e a gestão das APs, que por sua vez podem gerar informações fundamentais para a identificação de assets potenciais (assets que podem gerar outras formas de valor), incluindo capital humano existente, infraestrutura e arranjos institucionais. A coleta de documentos ocorreu entre abril de 2015 e janeiro de 2017, na plataforma online do Instituto Chico Mendes de Conservação da Biodiversidade. Nos planos de manejo, utilizaram-se as seguintes informações: a caracterização do ambiente (fatores bióticos e abióticos), o contexto em que está inserido, histórico, legislação, atividades desenvolvidas, aspectos institucionais (profissionais, infraestrutura, parcerias e acordos), planejamento e zoneamento.

Primeiramente, foram coletadas informações gerais sobre as APs, como: ecossistema, ano do documento, ano de criação do parque e área em hectares ${ }^{2}$. Em seguida, as informações dos planos de manejo foram sintetizadas, para posteriormente serem classificadas, a partir do reconhecimento de palavras e frases que sintetizavam uma categoria de asset, ou um valor inerente à presença de uma área protegida, ou que simplesmente pareciam ter relevância temática. Estas foram assinaladas ao longo do texto (cada asset foi destacado apenas uma vez em cada PM).

2 Este tipo de informação é útil para testar variáveis que influenciam tanto na presença como na ausência de assets nas unidades de conservação. Entretanto, sugerimos que sejam utilizadas quando a amostra for maior do que a que trabalhamos neste artigo, possibilitando uma avaliação estatística mais detalhada. 
Posteriormente, as palavras ou frases que haviam sido assinaladas ao longo do texto foram revisadas para a confirmação da presença do asset com base na lista de assets descrita acima. Para um asset ser reconhecido, é necessário responder ao menos duas das três perguntas-chave que norteiam a definição da proposta de assets de Caldecott \& Jepson (2014): i) as pessoas podem capturar valor desse asset no momento presente ou no futuro?; ii) esse asset é passível de receber investimento?; e iii) esse asset pode estar em risco?

Por fim, identificamos os dez assets que apareceram em um maior número de planos de manejo e discutimos também a presença e ausência de assets nos documentos, tendo em vista os aspectos paisagísticos e culturais das áreas úmidas globais, de importância internacional.

\section{Resultados e discussão}

Dos oito PARNAs em áreas úmidas identificados, sete tinham planos de manejo disponíveis na plataforma do ICMBio. São eles: Parque Nacional da Lagoa do Peixe, Parque Nacional de Ilha Grande, Parque Nacional do Araguaia, Parque Nacional do Cabo Orange, Parque Nacional do Pantanal Mato-Grossense, Parque Nacional do Viruá e Parque Nacional Marinho dos Abrolhos (Tabela 1). No momento da coleta de dados, o Parque Nacional de Anavilhanas apresentava ainda o documento de quando era uma estação ecológica, sendo, portanto, removido de nossa análise.

\section{Os assets nos parques nacionais de áreas úmidas}

Os sete planos de manejo dos parques analisados foram elaborados entre 1991 e 2014, e apenas dois relataram, na época, ter Conselho Gestor (Tabela 1). Essas APs foram criadas entre 1959 e 1998, com áreas variando entre 36.721,71 e 657.318,06 hectares, e abrangendo espacialmente todos os ecossistemas: Marinho Costeiro, Mata Atlântica, Amazônia, Pantanal e também áreas de transição com o Cerrado (Tabela 1).

Tabela 1 - Informações gerais dos parques nacionais em áreas úmidas do Brasil com planos de manejo.

\begin{tabular}{|l|l|c|c|c|r|}
\hline \multicolumn{1}{|c|}{ Parque } & \multicolumn{1}{|c|}{ Ecossistema } & Estado & $\begin{array}{c}\text { Ano de criação } \\
\text { da UC }\end{array}$ & Ano do PM & Área (hec) \\
\hline Lagoa do Peixe & Marinho Costeiro & RS & 1986 & 1999 & 34.400 \\
\hline Ilha Grande & Mata Atlântica & PR e MS & 1997 & 2008 & $78.251,89$ \\
\hline Araguaia & Cerrado, Amazônia, Pantanal (transição) & TO & 1959 & 2001 & 562.312 \\
\hline Viruá & Amazônia & RR & 1998 & 2014 & 216,427 \\
\hline Pantanal Matogrossense & Pantanal & MT & 1981 & 2003 & 135.000 \\
\hline Cabo Orange & Amazônia e Marinho & AP & 1980 & 2010 & 619.000 \\
\hline Abrolhos & Marinho Costeiro & BA & 1983 & 1991 & 91.300 \\
\hline
\end{tabular}

Um total de 230 assets distribuídos entre as cinco classes e suas subcategorias foram identificados nos documentos. Cada UC apresentou uma média de $32.9 \pm 8.2$ assets.

Os resultados mostram que os planos de manejo tendem a se concentrar em um número limitado de assets básicos, que se encaixam no paradigma institucional da conservação da biodiversidade (espécies de importância para conservação e espécies de importância econômica) e necessários para atividades, também básicas, de gestão (gestor/funcionário permanente; infraestrutura para gestão, eletricidade e veículos; e estratégias de planejamento e zoneamento) (Tabela 2). 
A representação particularmente alta de "beleza cênica" nos planos de manejo é bastante esperada em resposta aos objetivos de criação dos PARNAs brasileiros, definidos pelo Sistema Nacional de Unidades de Conservação (SNUC):

\begin{abstract}
"preservação de ecossistemas naturais de grande relevância ecológica e beleza cênica, possibilitando a realização de pesquisas científicas e o desenvolvimento de atividades de educação $e$ interpretação ambiental, de recreação em contato com a natureza e de turismo ecológico" (BRASIL 2000).
\end{abstract}

Assim, um foco em assets relacionados com a paisagem é esperado e, presumivelmente, representa o principal asset biofísico para a categoria "parque nacional". Esse resultado mostra que, pelo menos em um nível superficial, os parques nacionais são, em parte, definidos por suas belas paisagens, além de evidenciar que a relação dos homens com o ambiente natural tem sido vista predominantemente em termos biofísicos (Papayannis \& Pritchard 2008).

A beleza natural das paisagens de áreas úmidas, bem como a diversidade de vida (fauna e flora) - sobretudo de aves aquáticas - as tornam atrações ideais para atividades de recreação e turismo (Ramsar 2009b). No entanto, embora o asset relacionado às espécies, como "espécies com importância para conservação" esteja entre os dez assets mais recorrentes, assets relacionados ao turismo não foram bem representados. Um exemplo disso são assets humanos, como o turista em si e os guias, que podem melhorar a experiência do visitante, a infraestrutura necessária à sua recepção (asset de infraestrutura), entre outros - o que chama nossa atenção, especialmente porque um dos objetivos dos parques nacionais brasileiros é permitir as interações entre visitantes e natureza num contexto de ecoturismo.

Muitas das áreas úmidas com potencial paisagístico para o turismo já são parques nacionais, patrimônios da humanidade e sítios Ramsar - designações nacionais e internacionais compreendidas em nosso framework como assets institucionais. Essas áreas são capazes de gerar renda considerável através de seus atributos, como passeios de barco, pesca, observação de fauna e outros. Inclusive, em alguns países essas atividades representam um componente significativo na economia local e nacional (Ramsar 2009b).

Além, é claro, de que as áreas úmidas, suas paisagens e atributos, potencializam expressões artísticas, espirituais e culturais em todo o mundo (Ramsar 2009a), e esses valores são frutos de assets culturais como: fotografias, poemas, lendas, histórias, músicas, filmes e representação de religiões e divindades. No Brasil, onde há uma forte religiosidade, são muitas as divindades relacionadas à água, como Iemanjá, Oxum e Iara; personagens de religióes de matrizes africanas, como a Umbanda e o Candomblé. Em outras religiões ao redor do mundo, a água também tem um papel importante, como no Budismo, Cristianismo, Hinduísmo, Judaísmo e outros (Ramsar 2009a).

Entretanto, embora essas áreas sejam de grande importância cultural para a população humana, sobretudo no modo de vida de comunidades tradicionais (agricultura, provisão de água, madeira, pesca etc.), para turismo e recreação (já discutido aqui) e para o conhecimento, herança cultural (conhecimento tradicional, espiritualidade e crenças) e práticas sociais, como a pesquisa científica, educação e tradições culturais (Clarkson et al. 2013, Papayannis \& Pritchard 2008), a representação da categoria "assets culturais", nos 10 assets mais presentes, ficou por conta apenas dos programas e atividades de educação ambiental - propostos dentro dos objetivos, como citado anteriormente, corroborando com a colocação de Clarkson e colaboradores (2013), de que o valor educacional das áreas úmidas está intimamente alinhado aos benefícios para recreação $e$ bem-estar. 
Quadro A - Parque Nacional Marinho de Abrolhos e seus assets (assets destacados em negrito):

O PARNA abriga um complexo de recifes coralíneos, região com maior biodiversidade marinha do Atlântico Sul. Suas belas paisagens, cavernas submarinas, a presença das baleias Jubarte que vêm acasalar e dar à luz na região, suas aves marinhas, entre outros atributos, atraem turistas e pesquisadores do mundo todo para atividades como mergulho, observação de fauna e atividades científicas. Pronta para receber visitantes, o PARNA conta com um centro de visitantes, guias capacitados pelo ICMBio, trilhas e um conjunto de unidades de conservação em seu entorno (ICMBio 2018).

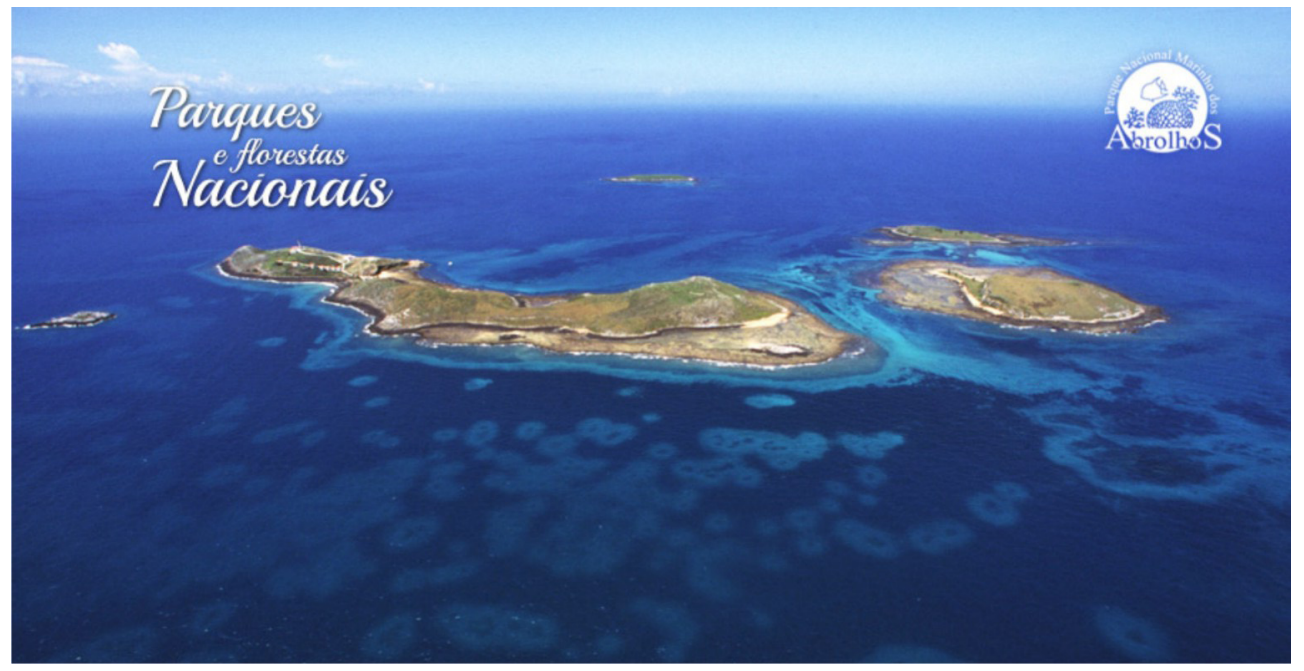

Figura 3 - PARNA Marinho de Abrolhos (Arquivo ICMBio - ICMBio.gov.br/parnaabrolhos).

Em relação à pesquisa, o baixo número dos assets como "pesquisadores" (asset humano) e "infraestrutura de pesquisa" (asset de infraestrutura) indica que são pouco reconhecidos nos PMs, o que contrasta com a premissa de que planejamento e decisões para o parque devem ser tomadas em consonância com resultados de pesquisas científicas. Ou seja, aparentemente a importância da pesquisa científica é reconhecida quando da formulação dos objetivos do parque, mas os planos de manejo ainda não deixam transparecer o valor que os parques podem gerar para os pesquisadores, e vice-versa. As áreas protegidas em geral, e os parques nacionais em particular, são importantes assets científicos/educativos nacionais, pois são áreas onde os processos ecológicos podem ser estudados (Correia et al. 2016), além de oferecer excelentes campos de pesquisa para uma variedade diversificada de disciplinas (Machlis \& McNutt 2015).

Uma publicação da Secretaria da Convenção Ramsar sobre os "liquid assets" das áreas úmidas, 40 anos depois da Convenção, mostra que o respaldo do tratado, com a adesão de novos países, as parcerias com organizações ambientais, os planos estratégicos e as conferências das partes contratantes são assets fundamentais e responsáveis pela evolução filosófica e política e pelo seu reconhecimento e difusão no mundo todo. Além disso, esses assets viabilizam financiamento a projetos e acordos/parcerias para gestão das áreas com organizações governamentais, regionais, organizações não governamentais e setores privados (Ramsar Convention Secretariat 2011). Entretanto, esses assets da classe institucional não tiveram tanto destaque em nossos resultados, que evidenciaram apenas os assets de "planejamento" e "zoneamento" dos PARNAs. Esses resultados sugerem que os planos de manejo de áreas úmidas brasileiras reconhecem, sobretudo, 
assets institucionais associados à gestão da área em si, mas não estão ainda reconhecendo o potencial institucional mais alargado dessas áreas, como parcerias comerciais ou internacionais e seus valores associados.

Embora os assets das áreas úmidas sejam fonte de múltiplos benefícios, muitas vezes não são reconhecidas ou apenas um ou poucos são apreciados (Russi et al. 2013). Argumentamos, portanto, que a ausência de um asset no plano de manejo pode ser devido a: i) o asset estar ausente no parque; ou ii) o asset estar presente no parque, mas não ser considerado relevante para a elaboração do plano de manejo. O primeiro é quase certamente o caso de subcategorias de assets como "monumentos históricos" e "associações de celebridades". O segundo caso são situações mais complexas, uma vez que o asset está presente, mas que poderá não estar sendo considerado para potencializar o valor da AP em questão, ou seja, por não ter sido reconhecida sua capacidade de gerar valor. São exemplos alguns assets culturais: marca/emblema baseado em um asset biofísico e lendas associadas aos PARNAs (ex. espécie de importância cultural, como peixe-boi), interpretações criativas (fotografias, exposições), eventos culturais (feiras e festivais típicos) e representações de mídia (livros, documentários, sites etc).

Tabela 2 - Os dez assets mais presentes nos planos de manejo dos parques nacionais em áreas úmidas do Brasil.

\begin{tabular}{|c|c|}
\hline Assets & UCs $(n=7)$ \\
\hline \multicolumn{2}{|c|}{ Biofísico } \\
\hline Beleza cênica (vistas) & 6 \\
\hline Espécies com importância para conservação & 7 \\
\hline Espécies com importância econômica & 6 \\
\hline \multicolumn{2}{|c|}{ Humano } \\
\hline Funcionários permanentes & 6 \\
\hline \multicolumn{2}{|c|}{ Infraestrutura } \\
\hline Propriedades/infraestrutura para gestão & 7 \\
\hline Veículos & 6 \\
\hline Eletricidade & 6 \\
\hline \multicolumn{2}{|c|}{ Institucional } \\
\hline Planejamento & 7 \\
\hline Zoneamento & 7 \\
\hline \multicolumn{2}{|c|}{ Cultural } \\
\hline Programas/Atividades de educação ambiental & 6 \\
\hline
\end{tabular}

\section{A abordagem de assets nos planos de manejo}

Muitas formas de se capturarem os valores de uma AP vêm sendo propostas e utilizadas no mundo, incluindo o desenvolvimento do turismo, via construção de cadeias produtivas e manejo comunitário, via pesquisa científica, via pagamento por serviços ambientais (Ferraro et al. 2011, Ferraro \& Hanauer 2014, Blare \& Donovan 2016, Campos-Silva \& Peres 2016, Campos-Silva et al. 2017). 
Nós argumentamos que o PA asset framework oferece uma abordagem complementar que pode fortalecer a gestão de áreas protegidas. Pois, muito embora a criação de AP seja a principal ferramenta global para conservação (Watson et al. 2014), um estudo recente sobre abundância de populações de aves aquáticas em áreas úmidas evidenciou que a conservação bem-sucedida dessas espécies depende principalmente de uma governança mais efetiva - o aumento da abundância observado em áreas protegidas estava associado apenas àquelas com governança eficaz (Amano et al. 2017). Enquanto que uma boa governança, por sua vez, está associada positivamente a investimentos em biodiversidade (Miller et al. 2012). Inclusive, uma publicação do The Economics of Ecosystems and Biodiversity (TEEB), para águas e áreas úmidas, conclui que a solução para esses ambientes está na compreensão dos valores e benefícios derivados das águas $e$ áreas úmidas fornecidos para a sociedade, transformando abordagens de gestão $e$ focando em um desenvolvimento econômico sustentável a partir do desenvolvimento e da implementação de políticas regionais, nacionais e internacionais que abordam seus assets (Russi et al. 2013).

Basicamente, levantar os bens/recursos/atributos/dimensões-chave que podem estar sendo negligenciados nos planos de manejo (e, consequentemente, pela gestão da $\mathrm{AP}$ ) irá possibilitar que os gestores e tomadores de decisão selecionem os assets prioritários a serem manejados ou investidos, considerando as demandas de conservação em nível nacional e internacional, e a realidade social e ecológica da AP em questão. Essa abordagem está em consonância com o inciso VIII, art. $2^{\circ}$ da Instrução Normativa n ${ }^{\circ}$ 7/2017/GABIN/ICMBIO, de 21 de dezembro de 2017, o qual prevê que o conteúdo do diagnóstico do plano de manejo da UC deve reconhecer recursos e valores fundamentais para o planejamento de uma UC, sendo diretriz fundamental no processo de elaboração e revisão de planos de manejo de unidades de conservação federais (BRASIL 2017):

\begin{abstract}
"Diagnóstico: análise e interpretação das informações contidas na caracterização da UC, contemplando a definição dos seus recursos e valores fundamentais, a avaliação de sua condição atual, a tendência $e$ as ameaças que a afetam, os quais subsidiarão a identificação das necessidades de dados e de planejamento para a gestão da UC."
\end{abstract}

Neste processo, um portfólio com os assets fundamentais para a AP, os valores que podem gerar e a identificação dos seus beneficiários, facilitará a construção do planejamento. A abordagem a partir de um framework de assets pode ser aplicada em qualquer categoria de áreas protegidas e, possivelmente, seria ainda mais benéfica para áreas de uso sustentável, onde o desenvolvimento de um conjunto diversificado de atividades sustentáveis é essencial para que a área protegida atinja seus objetivos relacionados ao desenvolvimento sustentável, fortalecendo a gestão e a capacidade de governança local - elementos fundamentais para o crescimento e desenvolvimento social (Amano et. al 2017). Entretanto, identificar os assets e compreender seus benefícios é apenas um primeiro passo, tendo em vista que a amplitude dos valores requer uma abordagem de tomadas de decisão eficazes e integradas (Russi et al. 2013).

\title{
Conclusão
}

Nossa abordagem constitui um novo ramo ainda em desenvolvimento dentro do arcabouço teórico das áreas protegidas. Os métodos de identificação dos assets podem ser utilizados para complementar planos de manejo e outras diretrizes de gestão das APs. Para que os assets possam ser identificados em sua totalidade, é importante incluir levantamentos em campo, diálogos e entrevistas com gestores, analistas, pesquisadores, moradores, turistas e demais usuários, de modo a compreender os múltiplos benefícios e para que todas as formas de valor possam ser acessadas. Assim, é possível ter informações completas para determinar as melhores ferramentas de captura dos valores por seus beneficiários. 
No entanto, em nossa amostra de planos de manejo, percebemos que o foco destes documentos ainda é em um modelo de conservação da biodiversidade, um modelo preservacionista, que não explora os potenciais dos parques para a população em geral.

Este estudo, portanto, surge como uma primeira etapa de uma abordagem que vem apresentar aos gestores de APs e à comunidade acadêmica da conservação da natureza outra possibilidade de pensar as áreas protegidas, a partir, inicialmente, da identificação de bens/recursos/atributos/ dimensões-chaves (assets) que devem ser prioritariamente investidos, seja em tempo ou recursos humanos e financeiros, de forma que esse investimento, em curto e longo prazo, gere retorno que otimize o trabalho de gestão da AP ou que proporcione desenvolvimento para a região.

\section{Agradecimentos}

Este projeto é fruto da pesquisa de mestrado da autora Norah Costa Gamarra, no Programa de Diversidade Biológica e Conservação nos Trópicos da Universidade Federal de Alagoas, intitulado: "O valor das áreas protegidas para além da conservação da natureza: identificação de assets em unidades de conservação federais".

Nossos agradecimentos à CAPES, pela concessão à bolsa de mestrado da autora; ao CNPq, pela concessão de bolsa de Professor Visitante Especial (PVE \#400325/2014-4) ao Dr. Paul Jepson e pelo financiamento do Projeto Universal (CNPq/MCTI n. 448966/2014-0) e FAPEAL/Institutional Links/Fundo Newton (FAPEAL \#60030 68/2018).

Agradecemos também aos colegas do Laboratório de Conservação no Século XXI, da UFAL, em especial à Bárbara Resende, e à $\operatorname{Prof}^{\mathrm{a}}$. Dra . Arrisete Costa, por suas valiosas contribuições.

\section{Referências bibliográficas}

Amano, T.; Székely, T.; Sandel, B.; Nagy, S.; Mundkur, T.; Langendoen, T.; Blanco, D.; Soykan, C. \& Sutherland, W.J. 2018. Successful conservation of global waterbird populations depends on effective governance. Nature, 553: 199-202.

Bragagnolo, C.; Gamarra, N.C.; Malhado, A.C.M. \& Ladle, R.J. 2016. Proposta metodológica para padronização dos estudos de atitudes em comunidades adjacentes às unidades de conservação de proteção integral no Brasil. Biodiversidade Brasileira, 1: 190-208.

Brasil, 1996. Decreto ${ }^{\circ} 1.905$, de 16 de maio de 1996. Promulga a Convenção sobre Zonas Úmidas de Importância Internacional, especialmente como Habitat de Aves Aquáticas, conhecida como Convenção de Ramsar, de 02 de fevereiro de 1971. Diário Oficial da União. Disponível em: http://www.planalto.gov.br/ ccivil_03/decreto/1996/D1905.htm. (Acesso em 20/01/2018).

Brasil, 2000. Lei $n^{\circ} 9.985$, de 18 de julho de 2000. Regulamenta o art. 225, § $1^{\circ}$, incisos I, II, III e VII da Constituição Federal, institui o Sistema Nacional de Unidades de Conservação da Natureza e dá outras providências. Diário Oficial da União. Disponível em: http://www.planalto.gov.br/ccivil_03/leis/19985. htm. (Acesso em 10/01/2015).

Brasil, 2017. Instrução Normativa $n^{\circ}$ 7/2017/Gabin/ICMBio, de 21 de dezembro de 2017. Estabelece diretrizes e procedimentos para elaboração e revisão de planos de manejo de unidades de conservação da natureza federais. Diário Oficial da União. Disponível em: http://www.icmbio.gov.br/portal/images/ stories/portarias/intrucao_normativa_07_2017.pdf. (Acesso em 21/10/2018).

Bardin, L. 1977. Análise de Conteúdo. Edições 70, Lda. 226p.

Berelson, B. 1952. Content Analysis in Communications Research. The Free Press. 220p.

Bernard, E.; Penna, L.A.O. \& Araújo, E. 2014. Downgrading, downsizing, degazettement, and reclassification of protected areas in Brazil. Conservation Biology, 28: 1523-1739. 
Blare, T. \& Donovan, J. 2018. Building value chains for indigenous fruits: lessons from camu-camu in Peru. Renewable Agriculture and Food Systems, 33: 6-18.

Börner, J.; Baylis, K.; Corbera, E.; Ezzine-de-Blas, D.; Honey-Rosés, J.; Persson, U.M. \& Wunder, S. 2017. The effectiveness of payments for environmental services. World Development, 96: 359-374.

Caldecott, B. \& Jepson, P. 2014. Towards a framework for Protected Area asset management. Smith Sch. Enterp. Environ. Univ. Oxford. 14p.

Campos-Silva, J.V. \& Peres, C.A. 2016. Community-based management induces rapid recovery of a highvalue tropical freshwater fishery. Scientific Reports, 6: 34745.

Campos-Silva, J.V.; Peres, C. A.; Antunes, A.P.; Valsecchi, J. \& Pezzuti, J. 2017. Community-based population recovery of overexploited Amazonian wildlife. Perspectives in Ecology and Conservation, 15(4): 266-270.

Chaikumbung, M.; Doucouliagos, H. \& Scarborough, H. 2016. The economic value of wetlands in developing countries: A meta-regression analysis. Ecological Economics, 124: 164-174.

Clarkson, B.R.; Ausseil, A.G.E. \& Gerbeaux, P. 2013. Wetland ecosystem services. In: Dymond J.R. (ed.) Ecosystem services in New Zealand: conditions and trends. Manaaki Whenua Press, Lincoln, 192-202.

Correia, R.A.; Malhado, A.C.M.; Lins, L.; Gamarra, N.C.; Bonfim, W.A.G.; Valencia-Aguilar, A.; Bragagnolo, C.; Jepson, P. \& Ladle, R.J. 2016. The scientific value of Amazonian protected areas. Biodiversity Conservation, 25: 1503-1513.

Correia, R.A.; Jepson, P.; Malhado, A.C.M. \& Ladle, R.J. 2018. Culturomic assessment of Brazilian protected areas: Exploring a novel index of protected area visibility. Ecological Indicators, 85: 165-171.

Ferraro, P.J.; Hanauer, M.M. \& Sims, K.R. 2011. Conditions associated with protected area success in conservation and poverty reduction. Proceedings of the National Academy of Sciences, 108: 13913-13918.

Ferraro, P.J. \& Hanauer, M.M. 2014. Quantifying causal mechanisms to determine how protected areas affect poverty through changes in ecosystem services and infrastructure. Proceedings of the National Academy of Sciences, 111: 4332-4337.

Foley, J.A. 2005. Global Consequences of Land Use. Science, 309(5734): 570-574.

Gamarra, N.C. 2017. O valor das áreas protegidas para além da conservação da natureza: identificação de assets em unidades de conservação federais. Dissertação (Mestrado em Diversidade Biológica e Conservação nos Trópicos). Universidade Federal de Alagoas. 124p.

Gamarra, N.C.; Correia, R.A.; Bragagnolo, C.; Campos-Silva, J.V.; Jepson, P.R.; Ladle, R.J. \& Malhado, A.C.M. 2019. Are Protected Areas undervalued? An asset-based analysis of Brazilian Protected Area Management Plans. Journal of Environmental Management, 249: 109347.

ICMBio (Instituto Chico Mendes de Conservação da Biodiversidade), 2018. O que fazemos: Parque Nacional Marinho de Abrolhos. Instituto Chico Mendes de Conservação da Biodiversidade. Disponível em: http://www.icmbio.gov.br/parnaabrolhos/o-que-fazemos.html. Acesso em: 20/10/2018.

Junk, W.J.; Piedade, M.T.F.; Lourival, R.; Wittmann, F.; Kandus, P.; Lacerda, L.D.; Bozelli, R.L.; Esteves, F.A.; Cunha, C.N.; Maltchik,L.; Schöngart, J.; Schaeffer-Novelli,Y. \& Agostinho, A.A. 2013. Brazilian wetlandas: their definition, delineation, and classification, for research, sustainable management, and protection. Aquatic Conservation: Marine and Freshwater Ecosystems, 4(1): 5-22.

Jepson, P.R.; Caldecott, B.; Schmitt, S.F; Carvalho, S.H.C.; Correia, R.A. \& Gamarra, N. 2017. Protected area asset stewardship. Biological Conservation, 212: 183-190.

Kleijn, D.; Cherkaoui, I.; Goedhart, P.W.; van der Hout, J. \& Lammertsma, D. 2014. Waterbirds increase more rapidly in Ramsar-designated wetlands than in unprotected wetlands. Journal of Applied Ecology, 51(2): 289-298. 
Ladle, R.J.; Jepson, P. \& Gillson, L. 2011. Social Values and Conservation Biogeography, p. 13-30. In: Ladle, R.J. \& Whittaker, R.J. (eds.) Conservation biogeography. Oxford University Press. 301p.

Machlis, G. \& McNutt, M. 2015. Parks for science. Science, 348: 1291-1291.

Mascia, M.B.; Pailler, S.; Krithivasan, R.; Roshchanka, V.; Burns, D.; Mlotha, M.J. Murray, D.R. \& Peng, N. 2014. Protected area downgrading, downsizing, and degazettement (PADDD) in Africa, Asia, and Latin America and the Caribbean, 1900-2010. Biological Conservation, 169: 355-361.

MEA (Millennium Ecosystem Assessment), 2005. Ecosystems and human wellbeing: wetlands and water. Synthesis reports. World Resources Institute, Washington, DC. 80p. Disponível em: https://www. millenniumassessment.org/documents/document.358.aspx.pdf. (Acesso em 22/01/2018).

Miller, D.C.; Agrawal, A. \& Roberts, J.T. 2013. Biodiversity, governance, and the allocation of international aid for conservation. Conservation Letters, 6(1): 12-20.

MMA (Ministério do Meio Ambiente), 2015. Recomendação CNZU n 7 de 11 de junho de 2015. Dispõe sobre a Definição de Áreas Úmidas Brasileiras e sobre o Sistema de classificação destas Áreas. Comitê Nacional de Zonas Úmidas (CNZU).

MMA (Ministério do Meio Ambiente), 2016. Áreas Úmidas - Convenção de Ramsar. <http://www. mma.gov.br/biodiversidade/biodiversidade-aquatica/zonas-umidas-convencao-de-ramsar $>$ (Acesso em 25/01/2018).

Oliveira, A.P.C. \& Bernard, E. 2017. The financial needs vs. the realities of in situ conservation: An analysis of federal funding for protected areas in Brazil's Caatinga. Biotropica, 49: 745-752.

ONU (Organização das Nações Unidas), 2005. The millennium development goals report. 75p. Disponível em: http://www.un.org/millenniumgoals/2015_MDG_Report/pdf/MDG\%202015\%20rev\%20 (July\%201).pdf. (Acesso em 21/10/2018).

Pack, S.M.; Ferreira, M.N.; Krithivasan, R.; Murrow, J.; Bernard, E. \& Mascia, M.B. 2016. Protected area downgrading, downsizing, and degazettement (PADDD) in the Amazon. Biological Conservation, 197: 32-39.

Papayannis, T. \& Pritchard, D.E. 2008. Culture and wetlands - a Ramsar guidance document, Ramsar Convention, Gland, Switzerland. 78p. Disponível em: https://www.ramsar.org/sites/default/files/documents/ library/cop10_culture_group_e.pdf. (Acesso em 21/10/2018).

Ramsar, 2009a. Factsheet 9: Recreation \& Tourism. Gland, Switzerland, Ramsar Convention Secretariat. Disponível em: http://archive.ramsar.org/pdf/info/services_09_e.pdf. (Acesso em 21/10/2018).

Ramsar, 2009b. Factsheet 8: Cultural values. Gland, Switzerland, Ramsar Convention Secretariat. Disponível em: http://archive.ramsar.org/pdf/info/services_08_e.pdf. (Acesso em 21/10/2018).

Ramsar Convention Secretariat, 2011. Ramsar's Liquid Assets. 40 years of the Convention on Wetlands. In Ramsar Convention Secretariate, Switzerland. Disponível em: https://www.ramsar.org/sites/ default/files/documents/pdf/Ramsar40_booklet/Ramsar_LiquidAssets_E.pdf. Acesso em: 21/10/2018.

Ramsar Convention Secretariat, 2013. The Ramsar Convention Manual: a guide to the Convention on Wetlands (Ramsar, Iran, 1971). 6th ed. Ramsar Convention Secretariat, Gland, Switzerland. 112p.

Ricciardi, A. \& Rasmussen, J.B. 1999. Extinction rates of North American freshwater fauna. Conservation Biology, 13: 220-222.

Russi, D.; ten Brink, P.; Farmer, A.; Badura, T.; Coates, D.; Förster, J.; Kumar, R. \& Davidson, N. 2013. The Economics of Ecosystems and Biodiversity for Water and Wetlands. IEEP, London and Brussels; Ramsar Secretariat, Gland. 78p.

Sala, O.E. et al. 2000. Global biodiversity scenarios for the year 2100. Science, 287: 1770-1774.

Swenson, J.J.; Carter, C.E.; Domec, J.C. \& Delgado, C.I. 2011. Gold mining in the Peruvian amazon: Global prices, deforestation, and mercury imports. PLoS One, 6(4): e18875. 
Symes, W.S.; Rao, M.; Mascia, M.B. \& Carrasco, L.R. 2016. Why do we lose protected areas? Factors influencing protected area downgrading, downsizing and degazettement in the tropics and subtropics. Global Change Biology, 22: 656-665.

Vucetich, J.A.; Bruskotter, J.T. \& Nelson, M.P. 2015. Evaluating whether nature's intrinsic value is an axiom of or anathema to conservation. Conservation Biology, 29: 321-332.

Watson, J.E.M.; Dudley, N.; Segan, D.B. \& Hockings, M. 2014. The performance and potential of protected areas. Nature, 515: 67-73.

Biodiversidade Brasileira - BioBrasil.

Número temático: Diagnóstico e manejo de áreas úmidas em áreas protegidas

$$
\text { n. 2, } 2019
$$

http://www.icmbio.gov.br/revistaeletronica/index.php/BioBR

Biodiversidade Brasileira é uma publicação eletrônica científica do Instituto Chico Mendes de Conservação da Biodiversidade (ICMBio) que tem como objetivo fomentar a discussão e a disseminação de experiências em conservação e manejo, com foco em unidades de conservação e espécies ameaçadas.

ISSN: 2236-2886 